DOI 10.15826/B978-5-7996-2935-9.11

ИССЛЕДОВАНИЕ ЭФФЕКТИВНОСТИ МЕТОДОВ ЧИСЛЕННОГО ИНТЕГРИРОВАНИЯ ДЛЯ БОРТОВОЙ МОДЕЛИ ДВИЖЕНИЯ КОСМИЧЕСКОГО АППАРАТА СИСТЕМЫ ГЛОНАСС

\author{
И. С. Загоруйко \\ Национальный исследовательский \\ Томский государственный университет
}

Произведено исследование трех методов численного интегрирования (Эверхарта, Адамса-Мультона-Бошфорта и коллокационного). В качестве параметра оценки производительности использовалось количество обращений к функции правых частей. Оценка точности получаемого решения происходила путем сравнения получаемого решения с эталонным.

\title{
RESEARCH OF EFFICIENCY OF NUMERICAL INTEGRATION METHODS FOR ON-BOARD MODEL OF MOTION TO GLONASS SYSTEM
}

\author{
I. S. Zagoruyko \\ National Research Tomsk State University
}

Three integrators (Everhart, Adams-Multon-Boschfort and collocation) for the orbital motion of the GLONASS spacecraft were investigated. The number of calls to the function of the right-hand sides was used as a parameter for evaluating performance. The accuracy of the obtained solution was evaluated by comparing the obtained solution with the reference one.

Целью работы является выбор эффективного метода численного интегрирования для использования его в бортовой модели движения космического аппарата (KА) системы ГЛОНАСС.

Космические аппараты системы ГЛОНАСС движутся по почти круговым орбитам под влиянием несферичности потенциала Земли и притяжения внешних тел - Луны и Солнца. При отсутствии управления в орбитальной динамике этих объектов наблюдается незначительный рост эксцентриситета орбиты, поскольку они захвачены [1]

(c) Загоруйко И. С., 2020 
в устойчивый резонанс Лидова-Козаи. Движение объектов является регулярным на значительных интервалах времени [1].

В работе рассматриваются три метода численного интегрирования [2]: метод Адамса-Мультона-Бошфорта (МАМБ), метод Эверхарта (МЭ) и коллокационный метод (KM).

Численный метод, используемый в бортовых алгоритмах, должен сочетать в себе два свойства: быстродействие и точность получаемого решения. Поэтому для решения задачи численного интегрирования уравнений движения КА системы ГЛОНАСС было проведено исследование, в котором были выявлены зависимость точности получаемого решения от порядка используемого метода и требуемой локальной точности, зависимость количества обращений к функции правых частей также от порядка метода и локальной точности.

Полученные результаты показывают, что МЭ и КМ близки по точности получаемого решения и быстродействию. Это объясняется тем, что МЭ является частным случаем KM, имея особую схему реализации; МАМБ превосходит МЭ и КМ по производительности, но имеет более низкую точность получаемого решения при равнозначной требуемой локальной точности для этих методов. Этот недостаток МАБМ можно обойти, повысив локальную точность. Количество обращений к функции правых частей при этом возрастет, но метод будет сравним по точности решения с МЭ и КМ.

\section{Библиографические ссылки}

1. Томилова И. В., Бордовицына Т. В. Особенности структуры резонансных возмущений неуправляемых объектов навигационных систем ГЛОHACC и GPS и их влияние на орбитальную эволюцию объектов // Изв. вузов Физика. - 2017. - Т. 60, № 4. - С. 119-125.

2. Авдюшев B. А. Численное моделирование орбит небесных тел.Томск : Издат. дом. ТГУ, 2015. - 335 с. 